\title{
Cinquantenaire de la trisomie 21
}

\section{Retour sur une découverte}

\section{Marthe Gautier}

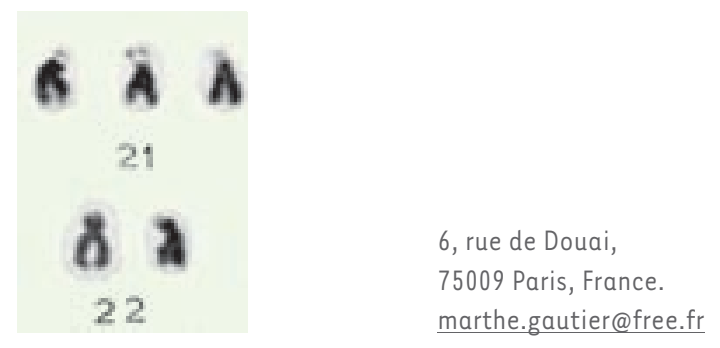

«En réalité, les découvertes sont dues à des personnes en marge des groupes de chercheurs formatés. »

Pierre Laszlo

II y a cinquante ans, j'étais cosignataire de l'article princeps qui établissait la présence d'un chromosome surnuméraire [1] dans le syndrome individualisé par Langdon Down en 1866 et communément appelé alors en France «mongolisme ». C'était la première aberration chromosomique autosomique reconnue dans les cellules de l'espèce humaine; elle reçut le nom de trisomie 21. II m'a semblé historiquement intéressant d'apporter mon témoignage personnel en tant qu'acteur de cette découverte.

\section{Rappel historique}

Se situer en 1958 implique de retrouver le contexte et les certitudes de l'époque. Alors qu'il était admis depuis plusieurs décennies que l'espèce humaine possédait 48 chromosomes, J.H. Tjio et A. Levan [2] démontrent en 1956 qu'il n'y en avait que 46. Ceci affecta peu de gens hormis quelques généticiens - et pendant longtemps le chiffre de 48 fut enseigné dans les écoles. L'étape qui paraissait simple fut suivie d'autres plus importantes nous entraînant vers la connaissance des origines de la vie; elle ne fit pas le même bruit médiatique que la mise en orbite du premier satellite artificiel, le Spoutnik (en russe, compagnon de voyage) quelques mois plus tard. Ce vol contribua à nous conduire vers l'origine de l'univers. La science avance différemment au hasard des disciplines.

${ }^{1}$ Par un lapsus calami que je n'ose interpréter, mon nom fut inscrit de façon erronée Marie Gauthier. L'erreur fut corrigée dans les publications ultérieures.
II avait fallu attendre 30 ans avant que ne soient transmises aux biologistes les lois génétiques des petits pois de Johan Mendel, ou frère Gregor au couvent des Augustins de Brno; nous sommes en 1900. La même année, Nettie Stevens met en évidence l'existence des chromosomes sexuels chez un coléoptaire [3]. Vers 1910, les travaux de Morgan sur la drosophile, cette providentielle mouche du vinaigre qui se reproduit très vite et présente des chromosomes géants, inaugurent les débuts de la cytogénétique [4]. S'il n'y avait eu l'attitude d'Alexis Carrel (Prix Nobel en 1912) pendant la période de l'Occupation [5], son expérience des cultures cellulaires eût pu être alors largement utilisée. Mais, une longue suite d'erreurs et d'échecs découragea les chercheurs. Et ce n'est qu'en 1949, et uniquement sur les cellules d'épiphyse de chat, que Barr et Bertram [6] découvrent l'existence d'un corpuscule dans le noyau des femelles; il s'agissait en fait d'un phénomène général qui signait la présence de deux chromosomes X. L'explication cytologique (lyonisation) en revint à Mary Lyon [7]. Le simple frottis de la muqueuse buccale permit alors d'aborder les diagnostics des états intersexués.

\section{La découverte de la trisomie 21, telle que je l'ai vécue...}

\section{Les prémices}

J'arrive à Paris, en 1942, en pleine guerre, près de ma sœur aînée Paulette, interne à l'Institut Gustave Roussy, en fin d'études de médecine. Elle m'initie aux arcanes du statut d'étudiante. Elle me met en garde: "Quand on est une femme, qu'on n'est pas fille de patron, il faut être deux fois meilleure pour réussir ». J'aborde le PCB (prépa de médecine) : facile. En 1944, Paulette est tuée par des Allemands en débâcle, au moment de 
la Libération. Pour mes parents, dans leur chagrin, dès ce moment, il a fallu que je sois à la fois elle et moi : difficile. Mon objectif, les concours qui ouvrent les portes. Après l'externat, je réussis l'internat des Hôpitaux de Paris (IHP), tant convoité et, à l'époque, peu féminisé (les femmes n'y ont eu accès qu'en 1885). Dans ma promotion, sur 80 internes nommés, il n'y a que deux filles.

Après quatre ans d'un merveilleux apprentissage clinique en pédiatrie, un de mes maîtres, le Pr R. Debré, pape de la pédiatrie, me propose une bourse d'un an à Harvard, offerte par un mécène qui venait de fonder la SESERAC². Objectif: la cardiologie infantile pour: (1) éradiquer la maladie de Bouillaud ou RAA (rhumatisme articulaire aigu) par la pénicilline et traiter les cardites parfois mortelles par la cortisone, encore peu disponible en France ; j'avais consacré ma thèse à l'étude clinique et anatomopathologique des formes mortelles de cette affection due à l'agression du streptocoque $\mathrm{A}$ bêta-hémolytique, germe resté très sensible à de faibles doses de pénicilline, qui n'était arrivée en Europe que tardivement, après la guerre ; (2) créer un département pour le diagnostic et la chirurgie des cardiopathies congénitales du nouveau-né et du nourrisson. Perspectives nouvelles et fascinantes: apprendre pour mieux soigner et guérir des enfants...

Après quelques hésitations, j'accepte, non sans réticences: quitter amour, amis, famille pour un an, sans se revoir, ni se téléphoner (trop coûteux à l'époque). Mais ma décision est prise: en septembre 1955, le trajet en train Paris-Le Havre se fait dans les larmes. On embarque la cantine sur le Mauritania de la compagnie Cunard (l'avion est bien trop onéreux pour des boursiers). Par chance, deux collègues amis IHP, pédiatres de l'école Robert Debré, Jean Aicardi et Jacques Couvreur ${ }^{3}$, boursiers Fullbright, sont du voyage et, par chance, aussi basés à Boston. Nous sommes les premiers IHP à bénéficier d'une bourse d'étude aux États-Unis.

Plus de cinq jours en mer; une toute petite tempête ; et lentement, au petit jour, l'arrivée. Les hélices se taisent doucement. Les gratte-ciel de Manhattan se découpent sur un ciel merveilleusement bleu. Nous sommes les hôtes de l'oncle Sam. Bien qu'imparfaitement bilingues, nous ne sommes pas des émigrés «sans-papiers ». Nous avons un visa d'un an.

\section{«As a pilgrim 》 I am in Boston}

Vingt-quatre heures pour trouver un appartement en co-location, acheter un lit, une chaise et une table aux puces locales. Le Pr David Rutstein a prévu un programme parfait : chez le Pr Alexander Nadas, pionnier du diagnostic des cardiopathies congénitales (CC) avant chirurgie et chez le Pr Benedict Massell, responsable du RAA. Je visiterai aussi plusieurs centres spécialisés sur le RAA: Cleveland, Chicago, San Francisco, Seattle, la Nouvelle Orléans, Washington. L'accord était en effet loin d'être fait sur la dose de cortisone à prescrire et sur la durée du traitement. On se demandait aussi si cette drogue «miracle » pouvait prévenir l'apparition

\footnotetext{
${ }^{2}$ Un de ses enfants venait de mourir de maladie de Bouillaud, faute de cortisone en France et il avait fondé la Société d'Etudes et de Soins pour Enfants atteints de RAA et de CC.

${ }^{3}$ Par la suite, Jean Aicardi fit une brillante carrière nationale et internationale en structurant la neurologie infantile; un syndrome porte désormais son nom. Quant à Jacques Couvreur, il partagea sa vie de pédiatre entre l'hôpital et la clientèle privée et fut le référent national dans le traitement des toxoplasmoses congénitales.
}

des cardites. Les responsables de chacun de ces centres m'ont fait partager leur expérience et leurs opinions, qui, dans leurs divergences même, m'ont beaucoup appris. Ce voyage sera effectué bravement, en solo, dans les autobus Greyhound (plus de dix nuits pour économiser les frais d'hôtel, mais les bus sont bien supérieurs aux avions pour apprécier le paysage...).

Un autre «job» m'est assigné que j'ignorais: technicienne dans le laboratoire de culture cellulaire (fragments d'aorte). C'est un plus. À temps partiel, à mon gré, éventuellement le dimanche. Qu'à cela ne tienne. Une charmante technicienne m'apprend tout ce qu'on doit connaître en culture cellulaire, et en plus elle m'initie au slang. Tout est à portée de main dans un congélateur. Je savais surveiller les cultures au microscope, les prendre en photos et développer celles-ci. Je constitue des dossiers pour les biochimistes qui font des études comparatives des taux de cholestérol dans les fibroblastes d'enfants et d'adultes. Je remplace la responsable du labo en congé de maternité. Je fais des séjours prolongés à la grande bibliothèque située à l'étage. J'explore les diverses techniques de cultures cellulaires, les données récentes en cardiologie. Mais à l'époque, rien sur la génétique ne m'a interpellée.

La bibliothèque est un lieu de rencontres et d'échanges. Nous, Français, sommes perçus comme issus d'un pays sous-développé, qu'il faut toujours aider à terminer ses guerres, et qui s'emmêle en Algérie! Depuis, je plains et défends tous les immigrés du monde.

Mon visa expire enfin. Retour sur le Flandres. J'ai honoré ma dette vis-à-vis de mon mécène et reviens pleine d'enthousiasme et de projets. J'arrive au petit jour au Havre. On m'attend sur le quai...

À Paris, il faut reprendre ses esprits

\section{Nouveau décor}

Le poste de chef de clinique promis chez le $\operatorname{Pr} M$. Lelong avant mon départ a été donné à un collègue en mon absence. Seuls postes disponibles: à l'hôpital Trousseau chez le Pr R. Turpin chez qui je n'ai jamais été ni stagiaire, ni externe, ni interne. Nous ne nous connaissons pas. Je ne suis pas une élève de la maison! Avec l'ami Jean Aicardi, qui revient lui aussi de Boston, nous voilà «chefs » en septembre 1956. Le clinicat est un poste d'enseignement à mi-temps, peu rémunéré, mais obligatoire pour monter vers l'assistanat et le médicat. L'atmosphère est celle d'un service hospitalier, figé dans sa hiérarchie typiquement française, et dont le patron est peu communicatif et très distant. Quel contraste avec l'atmosphère décontractée des États-Unis! Mais il faut «faire avec», avant de prendre son essor ailleurs et de gagner sa vie. 
Pédiatres avertis, nous savions que ce patron s'intéressait aux états malformatifs : tenter de distinguer l'inné de l'acquis. En 1937, il avait évoqué dans le mongolisme la possibilité d'une anomalie chromosomique proche de celle de la mutation Bar de la drosophile [8]. II n'était pas le premier, ni le seul à faire ce type d'hypothèse, mais n'était pas allé plus loin à ce jour. II s'était tourné vers l'étude des dermatoglyphes, un pis-aller de fortune, dans les recherches sur l'hérédité du mongolisme. En 1950, à Londres, L. Penrose [9] penchait plus pour une triploïdie que pour une trisomie ou une monosomie. Il a l'occasion d'avoir un échantillon testiculaire de patient qu'il confie à Ursula Mittwoch. La technique et les résultats sont incertains : elle conclut que les cellules ont « 47 ou 48 chromosomes», à une époque où le nombre normal dans l'espèce humaine était estimé à 48. Mais du moins la triploïdie était exclue.

\section{Le déclic}

Donc, à la rentrée 1956, le Patron, revenant du Congrès International de Génétique Humaine à Copenhague, nous apprend que le nombre de chromosomes de l'espèce humaine n'est pas de 48 , mais de «46»; il dit alors regretter qu'il n'y ait pas à Paris de lieux où faire des cultures cellulaires pour pouvoir compter les chromosomes des mongoliens. Surprise par cette remarque, je m'en étonne et, forte de mon expérience américaine, je propose « d'en faire mon affaire, si l'on me donne un local ». Je sais qu'il faut agir vite, sans se tromper, et réussir les premiers, car les équipes internationales vont ou sont déjà entrées en compétition, rivalité habituelle dans le domaine de la recherche, comme ailleurs. Je m'inscris en Sorbonne au certificat de biologie cellulaire. Je réalise qu'il ne faut pas compter sur l'aide des organismes de recherche, la France n'étant pas encore remise de la guerre, à peine en voie de restructuration avec I'INH ${ }^{4}$. Enfin, sciences et politique ne font bon ménage que quand finances il y a, ce qui n'est pas le cas. Le rôle de l'Université était celui de l'enseignement clinique ; elle n'était pas armée pour une recherche pointue. L'élite hospitalière n'avait pas encore compris qu'il lui appartenait de donner l'impulsion.

Un local vide est enfin mis à ma disposition, ancien labo de routine non utilisé. Trois pièces magnifiques, un frigidaire, une centrifugeuse, une armoire vide en haut de laquelle se trouve un microscope à faible définition. Eau, gaz, électricité. Et seule pour tout organiser, un rêve ! Je suis peu fortunée et aucun crédit de fonctionnement ne m'est proposé. C'est donc à mes frais, avec un emprunt,

${ }^{4}$ L'Institut national d'Hygiène avait été créé en 1941, et c'est seulement à partir de 1958 que des réformes sont entreprises. que je m'équipe en verrerie, appareil à eau distillée, etc. Aucun des produits nécessaires à la culture n'est commercialisé en France. Déterminée, je ne baisse pas les bras. Je prépare chaque semaine l'extrait embryonnaire frais, à partir d'œufs embryonnés de 11 jours que je vais chercher à l'Institut Pasteur. Pour le plasma, je ponctionne le sang d'un coq que j'ai acheté et qui est élevé dans un jardin à Trousseau. Et comme sérum humain, c'est le mien, procédé économique et sûr. Tout ceci a été exposé [10]. Je ne veux utiliser ni le poumon fœtal, ni les cellules de moelle osseuse, mais des explants de tissu conjonctif dont j'examine les cellules très jeunes in situ, en transplantant l'explant quand la pousse me paraît suffisante. Pas d'antibiotiques. Jamais de colchicine, car je redoute un éventuel effet néfaste sur l'intégrité du caryotype. Pas non plus de subcultures après trypsination, pour éviter des anomalies acquises in vitro par des cellules transformées. J'estime cela indispensable pour éviter tout artéfact, tel que chromosome erratique ou induit. Il faut faire preuve d'initiative, d'imagination et de discernement en cas d'échecs.

Ensuite, j'utilise - en l'adaptant - le principe du milieu hypotonique qui avait permis les résultats de Tjio et Levan [2], mais à base de sérum pour ne pas rompre la membrane cellulaire, enfin en laissant sécher les lames avant de les colorer [11]. Jamais de squash, préconisé par certains [12]. Ainsi, mes plus belles préparations sont en prométaphase, sans rupture de la membrane cellulaire, ce qui permet un chiffre exact et de très beaux chromosomes allongés, faciles à apparier, non cassés. Ces résultats ne sont acquis qu'après certains échecs. Je ne disposais d'aucune bibliographie, mais seulement de mes notes prises à Boston. Les témoins, que me procure le service de chirurgie voisin, proviennent d'interventions chirurgicales programmées chez des enfants normaux. Ils ont 46 chromosomes. J'ai maintenant deux techniciennes AP (Assistance Publique) qui, instruites par mes soins, sont remarquables ${ }^{5}$. Je leur transmets le doigté et l'expérience.

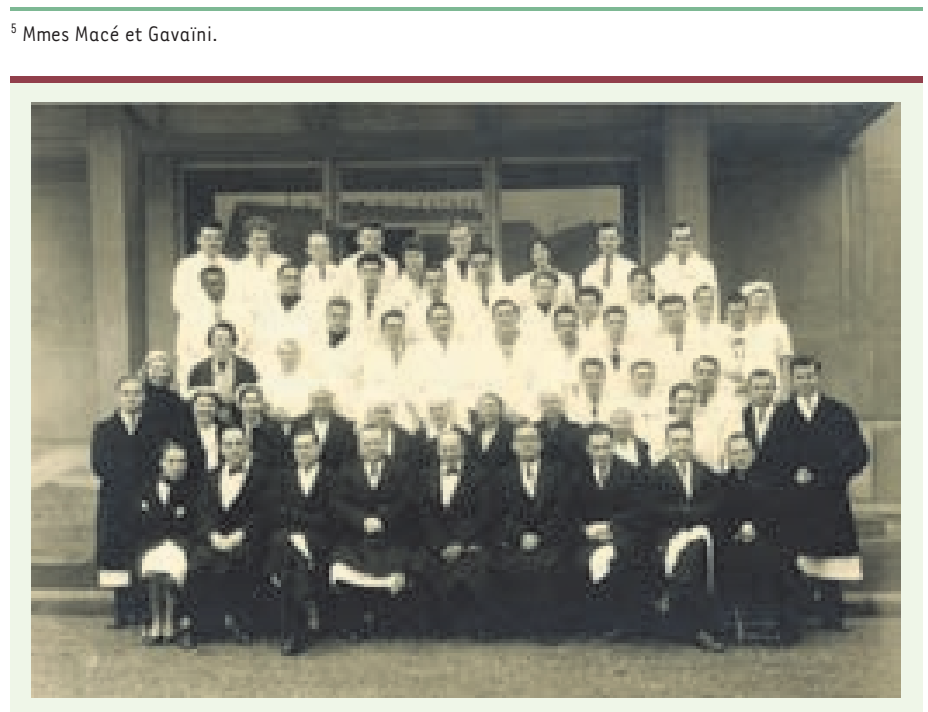

Figure 1. Service du professeur Turpin en 1957. Au premier rang; première à gauche, Marthe Gautier, troisième, Jacques Lafourcade, cinquième, professeur Raymond Turpin. Au second rang, premier à gauche, Jean Aicardi. 


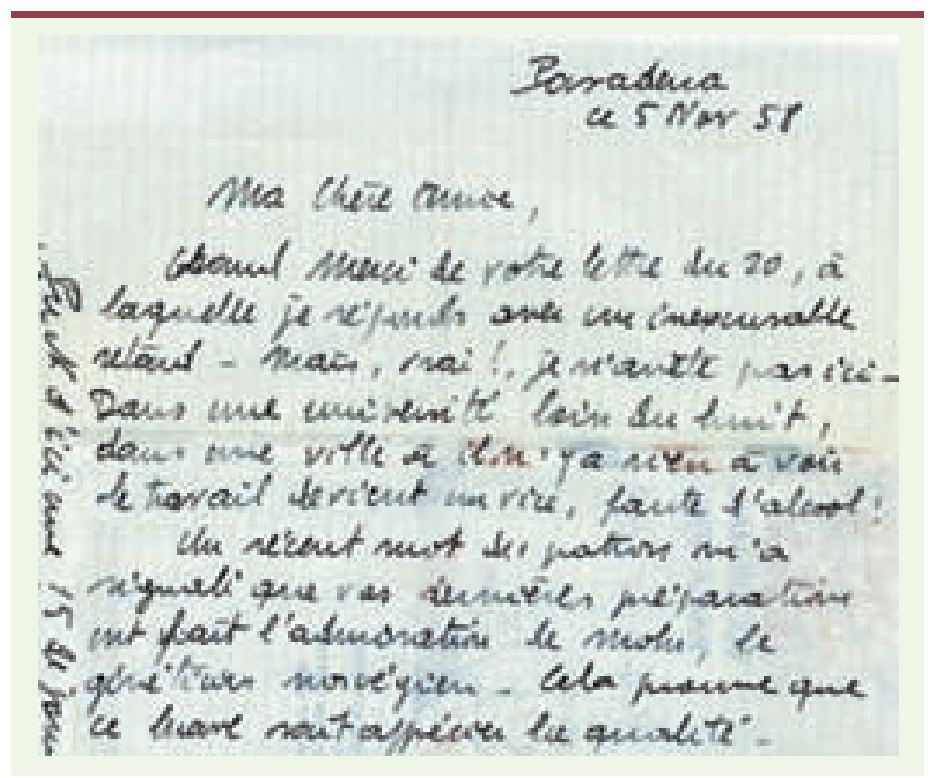

Figure 2. Photocopie d'une lettre envoyée par J.L. pendant son voyage aux États-Unis. II m'écrit «vos préparations... » c'est-à-dire les lames que j'avais obtenues avec les premières mitoses à 47 chromosomes.

\section{Un nouveau venu au laboratoire}

Je ne me souviens pas avoir eu au début de visites du patron. En revanche, son assistant Jacques Lafourcade passait me voir, un peu intrigué et au début sceptique sur l'éventualité d'une réussite de l'aventure, surtout dans les conditions précaires où elle était tentée. Sans doute rendait-il compte de l'évolution de mon travail.

Mais, je reçois bientôt des visites répétées de J. Lejeune. J.L., que je ne connaissais pas, était stagiaire au CNRS et élève du patron, comme en témoignent leurs publications communes sur les dermatoglyphes et l'effet néfaste des radiations ionisantes [13-16]. Je comprends vite l'intérêt qu'il porte aux cultures cellulaires, abandonnant sa loupe et ses statistiques sur la fréquence du pli palmaire médian.

Des tissus d'enfants mongoliens sont enfin obtenus ${ }^{6}$. En mitose, les cellules de mongoliens ont indiscutablement une différence: elles ont toutes 47 chromosomes, alors que tous les témoins en ont 46 . J'ai gagné mon pari, celui de réussir seule avec mes laborantines une technique et surtout de mettre en évidence une anomalie. C'est une découverte française. Cela n'était pas évident au départ.

Le chromosome supplémentaire est petit, le labo n'a pas de photomicroscope qui permettrait d'attester de sa présence et d'établir le caryotype. Je confie les lames à J.L. qui fait faire les photos, mais ne me les montre pas: elles sont, me dit-on, chez le Patron. Elles sont comme séquestrées. Ce chromosome ressemble au 21, mais il ne sera baptisé comme tel qu'à la conférence de Denver en $1960^{7}$.

${ }^{6}$ Je suis alors très occupée : j'ai en charge à mi-temps à l'hôpital Bicêtre le service de crèche de CC (cardiopathies congénitales), les consultations de RAA, et le début de ma clientèle privée.

${ }^{7}$ Ironie de l'histoire cytogénétique, après la classification à Denver en 1960 , on s'aperçut par la suite que ce chromosome était plus petit, donc correspondant à la $22^{\mathrm{e}}$ paire, mais tout resta dans cet ordre pour ne pas perturber la bibliographie déjà abondante sur le sujet.
Je suis consciente de ce qui se dessine sournoisement, mais n'ai pas assez l'expérience ni d'autorité dans ce milieu médical dont je n'ai pas encore compris les mécanismes pour savoir comment m'y confronter. Trop jeune, je ne connais pas les règles du jeu. Tenue à l'écart, je ne sais pourquoi l'on ne publie pas tout de suite. Je n'ai compris que plus tard que J.L., inquiet et n'ayant pas l'expérience des cultures, craignait un artéfact qui aurait brisé sa carrière - jusque-là assez peu brillante - mais qui, si les résultats étaient avérés, s'annonçait soudain géniale. Je soupçonne des manœuvres politiques... je n'avais pas tort. En revanche, personnellement, je n'avais pas l'intention «d'exploiter» ce chromosome surnuméraire, ma vie professionnelle se construisait ailleurs, vers la clinique.

Désormais, J.L. va se présenter comme le découvreur de la trisomie 21. Rapporteur CNRS au congrès des radiations ionisantes au Canada et, sans que cela soit prévu avec Turpin ni, bien sûr, avec moi, il parle de la découverte au séminaire McGill (en octobre 1958) comme s'il en était l'auteur. Je reçois cependant cette lettre datée du mois suivant pendant qu'il visite des labos aux États-Unis (Figure 2).

À ce moment, J.L. a été mis au courant des travaux de Patricia Jacobs qui venait de trouver dans le syndrome de Klinefelter un chromosome $X$ surnuméraire [17, 18]. Dès son retour, nous publions enfin, et cette fois en urgence, à l'Académie des Sciences, pour devancer inélégamment, en date, les équipes anglo-saxonnes [19]. Sans que j'aie pu encore voir les photos, sans avoir été informée de quoi que ce soit. Le texte m'est oralement communiqué un samedi midi pour être présenté le lundi. Exception française, car on pouvait, en effet, publier en trois jours aux CRAS (Comptes Rendus de l'Académie des Sciences) à Paris, alors qu'il fallait deux mois dans les journaux internationaux. Nous sommes donc les premiers à publier cette découverte dans le milieu scientifique international, après en avoir parlé au séminaire McGill. Contrairement à l'usage, J.L. signe en premier et mon nom ne figure qu'en second. Conformément à l'habitude, le Pr Turpin, chef responsable de l'hypothèse de départ, signe en dernier. Je suis blessée et soupçonne des manipulations, j'ai le sentiment d'être la «découvreuse oubliée ».

Un grand vacarme médiatique est alors orchestré par J.L. qui est interviewé par tous les journaux. Une grande découverte française... Dès lors, J.L. est couvert de récompenses; de stagiaire au CNRS, il est promu maître de recherche, médaille d'or. Sans avoir suivi le cursus universitaire, il sera ensuite nommé professeur de cytogénétique, poste créé par le $\operatorname{Pr}$ Turpin pour son élève. Cette chaire va ouvrir l'ère de la cytogénétique en France, tandis que cette discipline se développe partout dans 
le monde et que s'étend la renommée de J.L. II reçoit le prix Kennedy, sans demander que j'y sois associée. Progressivement et en participant à de nombreux congrès, il se présente comme le seul découvreur et finit par s'en convaincre, à tel point que les héritiers du Pr Turpin s'en émurent juridiquement par voie d'avocat. Ils déposèrent en outre dans les archives de l'Institut Pasteur les articles de leur père attestant son antériorité dans l'hypothèse chromosomique du mongolisme, enfin vérifiée. Pourtant, désormais, «le père de la trisomie 21 » comme le présentent désormais les médias, devient une sorte de thaumaturge $^{8}$ dont les tentatives de traitement de la trisomie 21 laissent sceptiques de nombreux scientifiques, car elles ne reposent pas sur des mécanismes biochimiques crédibles. Par la suite, et dans une continuité très cohérente, à la fin des années 1960, le diagnostic prénatal devient possible ; puis, en 1975, est votée la loi sur l'avortement qui va susciter chez J.L., croyant intransigeant, indignations, batailles et polémiques. Elles attiseront de graves querelles dans la société et provoqueront un réel désarroi parmi les cytogénéticiens dont certains souhaitaient que le diagnostic prénatal soit pratiqué en France. À ce moment, comme le mentionne André Boué , le comité Nobel aurait envisagé de récompenser la découverte de l'origine du mongolisme. Est-ce en raison de ses prises de position que le prix Nobel n'a pas été attribué à Jérôme Lejeune, seul nom qu'avaient fait résonner les trompettes de la renommée?

\section{Épilogue}

Première anomalie autosomique humaine ou première anomalie gonosomique, à Paris ou à Edinburgh, ces trouvailles furent faites de façon concomitante, comme c'est souvent le cas quand sont atteints les niveaux scientifiques et technologiques. Si je n'avais été la première, d'autres y seraient parvenus. Quoi qu'il en soit, je n'ai gardé aucun souvenir agréable de cette période, tant je me suis sentie flouée à tous égards. Mais dans l'histoire des «découvrances», combien d'autres furent ainsi passés sous silence, comme le Bâlois Johann Friedrich Miescher ou la Britannique Rosalind Franklin, pour ne parler que de l'ADN.

Depuis, la génétique moléculaire a vite rattrapé et dépassé la cytogénétique. Nous connaissons à présent la carte physique du chromosome 21 avec 225 gènes dont 127 seulement sont aujourd'hui identifiés - et sa séquence, réalisée en l'an 2000.

\footnotetext{
${ }^{8}$ Personne qui fait ou prétend faire des miracles.

${ }^{9}$ Cf. Entretien André Boué, janvier 2001. Histoire de l'Inserm : http://infodoc.inserm. $\mathrm{fr} /$ histoire
}

Depuis, également, la considération pour les femmes scientifiques a sans doute progressé, puisqu'en 2008, le prix Nobel a été décerné non seulement à Luc Montagnier, mais aussi à Françoise Barré-Sinoussi pour leurs travaux sur la découverte du rétrovirus responsable du sida [20]. Un espoir pour l'avenir. $\diamond$

\section{SUMMARY}

Fiftieth anniversary of the trisomy 21 : return on a discovery

Fifty years ago, I was co-author of the first paper asserting the presence of a supernumerary chromosome in Down's syndrome (called «mongolism» in France at that time). This first autosomal chromosomal abnormality was called Trisomy 21 . It seemed to me historically interesting to bring my own testimony as an actor in this discovery. $\diamond$

\section{REMERCIEMENTS}

Je tiens à remercier Joëlle Boué et Simone Gilgenkrantz qui m’ont encouragée à faire resurgir ces très anciens souvenirs.

\section{RÉFÉRENCES}

1. Lejeune J, Gauthier Marie, Turpin R. Les chromosomes humains en culture de tissus. CR Hebd Seances Acad Sci (Paris) $1959 ; 248: 602-3$.

2. Tjio HJ, Levan A. The chromosome number of man. Hereditas $1956 ; 42: 1-6$.

3. Gilgenkrantz S. Nettie Maria Stevens (1861-1912). Med Sci (Paris) 2008 ; $24: 874-8$.

4. Morgan TH, Bridges CB, Sturtevant AH. The genetics of Drosophila. Bibliographica Genetica $1925 ; 2: 1-262$.

5. Gilgenkrantz S, Rivera EM. The history of cytogenetics. Portraits of some pioneers. Ann Genet $2003 ; 46: 433-42$.

6. Barr ML, Bertram EG. A morphological distinction between neurones of the male and female, and the behaviour of the nucleolar satellite during accelerated nucleoprotein synthesis. Nature $1949 ; 163: 676$.

7. Lyon MF. Gene action in the X-chromosome of the mouse (Mus musculus L.). Nature 1961 ; $190: 372-3$.

8. Turpin R, Caratzali A, Rogier H. Étude étiologique de 104 cas de mongolisme et considérations sur la pathogénie de cette maladie. Premier congrès de la Fédération internationale latine des sociétés d'eugénisme, Paris: Masson, 1937, 154-64.

9. Penrose LS. Value of genetics in medicine. Br Med J $1950 ; 2: 903-5$.

10. Lejeune J, Turpin R, Gautier M. Étude des chromosomes somatiques. Technique pour la culture de fibroblastes in vitro. Rev Fr Etudes Clin Biol 1960; 5 : 406-8.

11. Rothferls KH, Siminovitch L. An air-drying technique for flattening chromosomes in mammalian oells grown in vitro. Stain Technology $1958 ; 33: 73-7$.

12. Hsu TC, Pomerat CM. Mammalian chromosomes in vitro. A method for spreading the chromosomes of cells in tissue. J Hered $1953 ; 44: 23-9$.

13. Turpin R, Lejeune J. Analogie entre le type dermatoglyphique palmaire des singes inférieurs et celui des enfants atteints de mongolisme. CR Hebd Seances Acad Sci (Paris) 1954 ; $238: 395-7$.

14. Turpin R, Lejeune J, Génin Mlle, Lejeune B Mme. Recherche d'une éventuelle association entre I'hémophilie et certaines particularités des dermatoglyphes. Semaine des Hôpitaux de Paris 1955 (Annales de la Recherche Médicale, $\mathrm{n}^{\circ} 3$ ).

15. Turpin $\mathrm{R}$, Lejeune J. Effets probables de l'utilisation industrielle de l'énergie atomique sur la stabilité du patrimoine héréditaire humain. Bull Acad Natl Med 1955 ; 139 : $104-6$.

16. Turpin R, Lamy M, Bernard J, Lefebvre J, Lejeune J. Nécessité de limiter l'exposition aux radiations ionisantes. Arch Fr Pediatr 1957 ; 14 : 1055-6.

17. Jacobs PA, Strong JA. A case of human intersexuality having a possible XXY sex-determining mechanism, Nature $1959 ; 183: 302-3$.

18. Harper P. The beginnings of human cytogenetics. Paris : Scion, $2006: 200 \mathrm{p}$.

19. Jacobs PA, Baikie AG, Court Brown WM, Strong JA. The somatic chromosomes in mongolism. Lancet $1959 ; 1$ (7075) : 710 .

20. Costagliola D. Prix Nobel de Médecine 2008 (Françoise Barré-Sinoussi et Luc Montagnier): Une vie consacrée à combattre le Sida. Med Sci (Paris) 2008; $24: 979-80$.

\section{TIRÉS À PART}

M. Gautier 

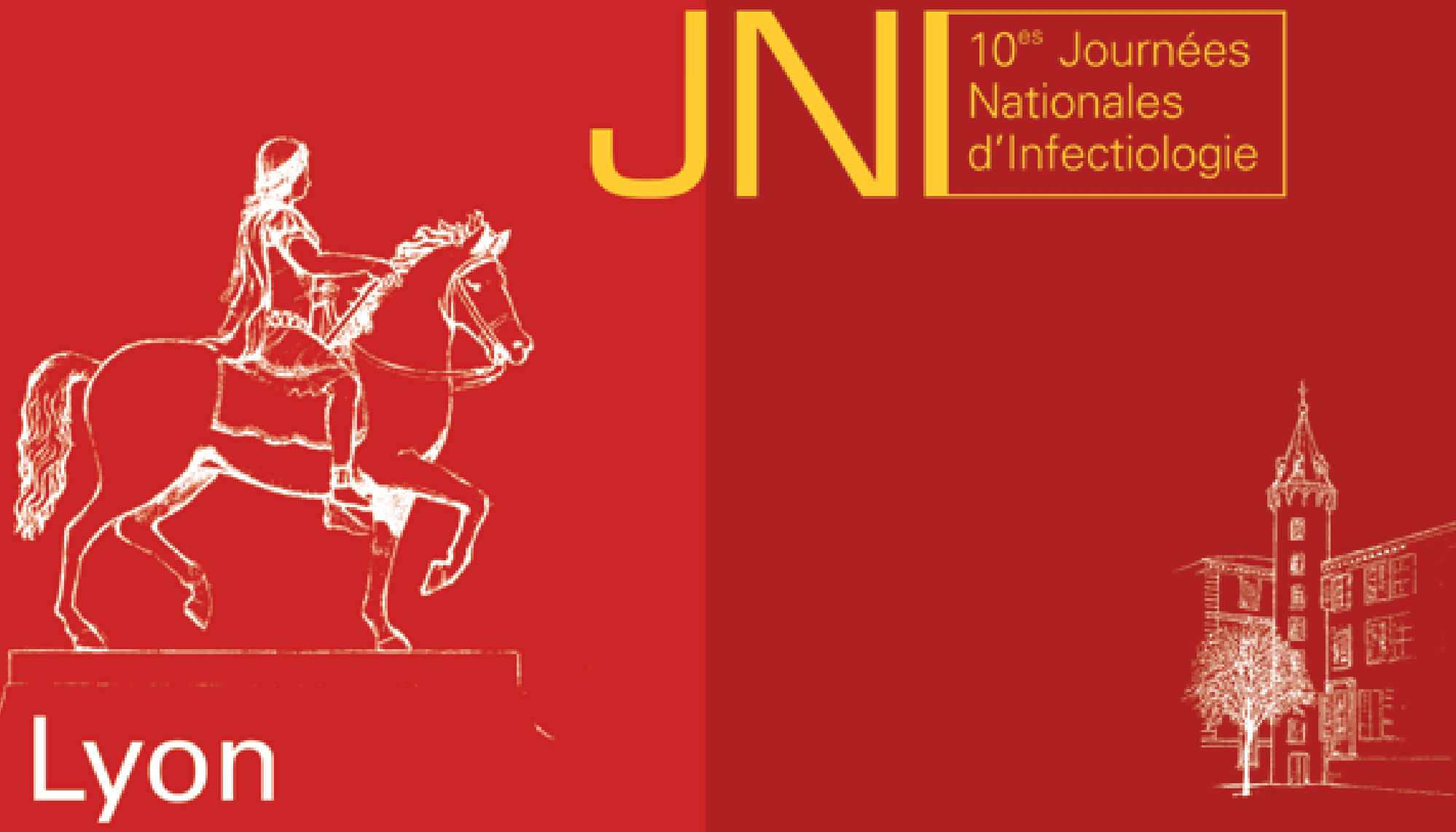

Du mercredi 10 juin, 13 h45 au vendredi 12 juin 2009, 15 h00

Cité Centre de Congrés - Lyon, Cité internationale, 50 quai Charles de Gaulle, 69463 Lyon Cedex 06

\section{Programme préliminaire}

- Conférence plénière - Mercredi 10 juin 2009 Génomique et pharmacogénetique dans le domaine du ViH

- Sessions de communications orales thematiques

"Best of" en indectiologie.

Bonnes pratiques cliniques

- Deisuner "Recherche"

Paludisme : actualiés et perspectives

VIH : les nouvelles voies de la prévention

VIH : la recherche trancaise dans les pays du

Nond et les pays du Sud : quelques exemples

Intectiologie, innovation therapeutique, contrainses téglementaires et économiques : quel avenir ?

- Ateliers

Candidoses imvasives : nouveles options

therapeutiques

Cas cliniques en médecine tropicale (FMC)

Vaccinaticns des adultes (FMC)

Viellissement accélére et infection VIH:

quelles consbquences cliniques, quelle prise en charge ? (FMC)

Depistage de l'infection a VH

Gestion drune stoution epidemique a enoerocoques ressistant à la vancomvcine (FMC)
- Sessions de communications orales en partenariat avee :

- le Centre National de Ráference des Enserovitus

- ie Centre National de Refference

des Staphylocoques

le GPIP (Groupe de Pathologie Intectieuse

Pedastriquel

- IINPES (Institut National de Prevention

et déducation pour la Santes)

- Iinvs institut de Veille sanitaire

- I'Onerba (Observatoire National

de l'Épidémiologie de is Resistance

Bactérienne aux Antibiotiques)

le RTRA Finovi (Fondation Innovations

en Infectiologie)

- la SFAR (Sociaté Francaise d'Anesthesie

et de Reanimation) (FMC)

la SFHH (Socithe Française d'Hygiene

Hospitalière) (FMC)

- Sessions de communications orales libres

- Sessions de posters discutés

- Posters

- Posters mysteres

- Symposiums integres

- Atelier intégré

\section{4* Journée des référents} en Antibiothérapie

Mereredi 10 juin 2009, 9h15 à $16 \mathrm{~h} 00$ Cité Centre de Congrès - Lyon

\section{Journée Nationale}

des Infirmièr(e)s en Infectiologie Jeudi 11 juin 2009, 8h45 à 17h15 Cité Centre de Congrès - Lyon

\section{www.infectiologie.com}

\section{NOLVEAU}

Inscription uniquement en ligne !

Cocrdination logistique

Alinea+ Communications $/$ Vivactis Plus

17 rue Jean Daudin. 75015 Paris

THL ; + 33 (0)1 $43374015-$ Fax : + 33100143376503 E-mail : vivactis Ovivactisplus com
Thenident do Cengits

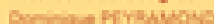

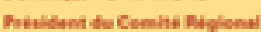
rongentation

Coviatian Oedork

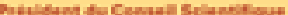
Micher Dupcis

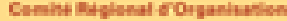

Poynce ADE Milspe sevintuor Jem atriout Jempud Buion Jenperie Bay Alerendraceiver

$$
\begin{aligned}
& \text { Arne Fntsuan } \\
& \text { Milope cravit } \\
& \text { Aneste concuot } \\
& \text { Nacale Ltcuraco } \\
& \text { finters wucit }
\end{aligned}
$$

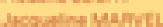

Cenaed Stientifleve

Loun atmuno Tanpoit CAMON

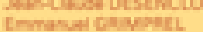
trmanui claver Deder way
Francia Prrinok Jean Fivistave hem Winetco tommanulie valio Doman PLNOENT alpes valestars 